of Natal Press, 1994. VIII, 319 S.: Ill., graph. Darst., überw. Kt. ISBN 0-86980-895-8/0-86980-894-X, DM 90,-.

Johnson, Howard L.: Atlas of Oklahoma climate. Norman, Okla.: Univ. of Oklahoma Press, 1994. XII, 32, [104] S.: überw. graph. Darst. und Kt. ISBN 0-8161-26892, DM 90,-.

Few, Roger: Atlas der Naturlandschaften: die letzten Paradiese unserer Erde. München: Frederking \& Thaler, 1996. 240 S.: überw. Ill., graph. Darst., Kt. ISBN 3-89405343-7, DM 49,80.

Harris, Godfrey: Mapping Russia and its neighbors: the new (atlas) of the changed geographical face of the former Soviet Union. Los Angeles, Calif.: Americas Group, 1993. 13 S. + 1 Übersichtskt. und 6 Transparentfolien, ISBN 0-935047-14-X, DM 48, 10.

Ciolkosz, Andrzej; Marek Ostrowski: Atlas zdjęć satelitarnych Polski. Instytut Geodezji i Kartografii ... Warszawa: SCI \& Art, 1995. $130 \mathrm{~S}$.; überw. Ill. und Kt. ISBN 83-902261-1-1, DM 80,75.

Jouris, David: All over the map: an extraordinary atlas of the United States; featuring towns that actually exist! Berkeley, Calif.: Ten Speed Press, 1994 [erschienen 1995]. 96 S.: zahlr. Kt. ISBN 0-89815649-1, DM 26,98.

Schmidt, Karl J.: An atlas and survey of South Asian history. Armonk, NY.: Sharpe, 1995. XV, 168 S.: zahlr. Kt. ISBN 1-56324-334-2/156324-333-4, DM 58,-.

Osborne, Roger: The historical atlas of the earth: a visual exploration of the earth's physical past. London: Viking, 1995. 192 S.: zahlr.
Ill. und Kt. ISBN 0-670-85374-7/06708-5374-7, £ 21,40.

Johnson, Hugh: Der neue Weinatlas: Länder, Lagen, Qualitäten, Trauben, Traditionen, Produzenten, Etiketten. 26., Aufl., 374. - 388. Tsd. - Bern: Hallwag, 1995. - 320 S.: zahlr. Ill. und Kt. ISBN 3-44410428-6, DM 138,-

Bus + Trucker Atlas: D: [schneller und sicherer ans Ziel; mit über 60000 Spezial-Informationen, Deutschland 1:250000, Ballungs-/ Wirtschaftsraum-Detailkarten mit übersichtlichen Orientierungs- und Zufahrtshilfen für Stadt-, Gewerbe-, Industrie- und Fracht-Zentren 1:75000, grafische Autobahnkarten für besseren Überblick, umfangreicher Serviceteil]. Nürnberg: BMA GmbH, 1995. 440 S.: Ill., überw. Kt. DM 278,--

Road atlas of South Africa: [enlarged areas of interest, town plans of major centres, scenic routes highlighted] 2. impr. London: New Holland, 1995. 72 S.: zahlr. Ill. und Kt. ISBN 1-85368-393-0, DM 24,80 .

Atlas de la Russie et des pays proches... - Montpellier, 1995. 208 S.: zahlr. Kt. (Collection Dynamiques du territoires; 15), ISBN 2-11003428-9, DM 88,-.

Historical atlas of the Holocaust / United States Holocaust Memorial Museum. New York, NY: Macmillan, 1996. 252 S.: überw. Kt. US \$ 40,61.

Shanks, Thomas G.: The international atlas: world longitudes \& latitudes, time changes and time zones. Rev. 3. ed. San Diego, Calif.: ACS Publ., Inc., 1991. 426 S. ISBN 0-935127-16-X, DM 56,57.

Lothar Zögner, Berlin

\section{Kartographie im Internet}

Eine Meta-Seite mit Informationen über und zur Kartographie gibt es in der Schweiz. Sie bietet Links zu kartographischen Institutionen, Behörden, kartographischen Verlagen, Firmen oder auch Kartensammlungen, vor allem in der Schweiz, aber auch weltweit, und behandelt das eidgenössische Kartenbibliothekswesen. Außerdem werden Suchinstrumente für Karten und raumbezogene Daten im Internet aufgeführt. Hinzu kommen Veranstaltungshinweise. Aus Sicht der Nutzer dürfte besonders wertvoll sein, daß für die Schweizer Links Vollständigkeit angestrebt wird. Die Seite wird von der ETH-Bibliothek (Dr. Jürg Bühler) und der Arbeitsgruppe Kartenbibliothekare (Dr. Thomas Klöti) betreut. Zur Zeit sind eine deutschsprachige und eine englische Version abrufbar:

< http://www.maps.ethz.ch >

Weiterte Anschriften:

$\square$ Schweizerische Gesellschaft für Kartographie (SGK)

http://www.geod.ethz.ch/karto/sgk/ start_sgk.html

$\square$ 47. Deutscher Kartographentag http://www.tu-dresden.de/fghgik/ $\mathrm{kt} /$ frame.html

$\square$ Arbeitsgruppe „Kartennutzung“ der DGfK

http://www.kfunigraz.ac.at/geowww/ ak.html

$\square$ Arbeitsgruppe „Hochgebirgskartographie" der DGfK http://www.geod.ethz.ch/karto/ hgkarto/ak2.html

$\square$ Arbeitsgruppe Kartographiehistoriker "D-A-CH“ http://biblio.unibe.ch/dach/index html

\title{
Veranstaltungen 1998
}

März 98 DGfK-Seminar „Multimediale Kartographie“ 1997198, Teil U, Ruhı Universität Bochum, Geographisches Institut (s. KN 4/97, S. 157)

6.-9.5. GeoBIT. Internationale Fachmesse für raumbezogene Informationstechnologie und Geoinformatik, Leipzig

1.-5.6. 47. Deutscher Kartographentag in Dresden. Leithema: „Kartographie, Kommunikation, Kunst". Die Fachvorträge werden von einem umfangreichen Rahmenprogramm aus Besichtiguagen, Exkursionen u.a. begleitet (s. KN 4/97, S. 156-158, KN 5/97, S. 203).

30.9.-2.10. 9. Kartographichistorisches Colloquium in Rostock und Schwerin. Informationen durch: Prof. Dr. Gyula Pápay, Ablbeckerstr. 14, D-53639 Königswinter 